\title{
Optimization of Photovoltaic Energy Production through an Efficient Switching Matrix
}

\author{
Pietro Romano, Roberto Candela, Marzia Cardinale, Vincenzo Li Vigni, Domenico \\ Musso, Eleonora Riva Sanseverino \\ DEIM, University of Palermo \\ e-mail: pietro.romano@unipa.it
}

Cite as: Romano, P., Candela, R., Cardinale, M., Li Vigni, V., Musso, D., Riva Sanseverino, E., Optimization of photovoltaic energy production through an efficient switching matrix, J. sustain. dev. energy water environ. syst., 1(3), pp 227-236, 2013, DOI: http://dx.doi.org/10.13044/j.sdewes.2013.01.0017

\begin{abstract}
This work presents a preliminary study on the implementation of a new system for power output maximization of photovoltaic generators under non-homogeneous conditions. The study evaluates the performance of an efficient switching matrix and the relevant automatic reconfiguration control algorithms. The switching matrix is installed between the PV generator and the inverter, allowing a large number of possible module configurations. PV generator, switching matrix and the intelligent controller have been simulated in Simulink. The proposed reconfiguration system improved the energy extracted by the PV generator under non-uniform solar irradiation conditions. Short calculation times of the proposed control algorithms allow its use in real time applications even where a higher number of PV modules is required.
\end{abstract}

\section{KEYWORDS}

PV systems, Photovoltaic modules, Mismatch, Optimization algorithm, Reconfiguration, MPPT, Dynamic electrical scheme

\section{INTRODUCTION}

The benefits brought by photovoltaic power generation include limited environmental impact and reduced installation costs, with the added incentive of government subsidy in many countries. Furthermore, photovoltaic modules are usually the lowest cost method of supplying electrical energy to remote, off-grid, low-power applications. The literature on the subject mostly deals with the problem of catching the maximum output power from these systems. In many papers, devices achieve the objective of maximizing output power by means of suitable conditioning systems that adapt the load to the generation system. This is carried out by Maximum Power Point Tracking (MPPT) algorithms [1-6] implemented on the inverter. Many solutions based on this methodology have been presented in the past few decades. In theory, the ideal operation is to determine the Maximum Power Point (MPP) of the photovoltaic (PV) array directly rather than tracking it by using the active operation of trial and error, which causes undesirable oscillations around the MPP (perturbation and observation). Other authors have proposed PV array or modules reconfiguration technique to improve the PV generation system's performance [7-11]. The fact that a higher efficiency can be attained by reconfiguration was proved in $[12,13]$. In [12] the issue of non-homogeneous solar irradiation was considered, but only a few configurations were explored and no automatic optimization algorithm was implemented for larger systems. In [13] a Matlab simulation of an automatic array reconfiguration technique, based on irradiance equalization, is presented. However the simulated architecture would only allow a few possible connections. The 
system is designed to improve performances of the PV generator taking into account fixed obstacles only. Mismatching and reconfiguration is also considered in depth in [7], where the effects of non-uniform solar irradiation distribution on energy output of different interconnected configurations in photovoltaic (PV) arrays is analysed. In [14] a suitable model taking into consideration the effects of bypass diodes and equivalent circuit parameters variation with respect to operating conditions is proposed. In [8] an evaluation of irradiation losses due to partial shadowing is given. In [15] the authors have proposed the use of a fixed matrix dimensions PV interconnection array, while in [16] Irradiance Equalization is implemented by a Dynamic Photovoltaic Array (IEq-DPVA) able to alter its matrix dimensions; the proposed algorithm supports exact row sizing thus each row maintains the same number of PV modules. In [17-19] solar cells from a solar adaptive bank are connected to the fixed part of a solar array. The self-adaptive reconfiguration method can result in rows with different numbers of solar cells. Finally, in [20] a Flexible Switch array Matrix topology is proposed to maximize the generated power in real-time under several mismatching conditions.

In this paper, the authors propose a new and efficient system for the maximization of the power output generated by the PV system by means of a suitable reconfiguration of the modules. In the following sections, the proposed system is described and optimization algorithms are explained and compared; finally, in the simulation section, the relevant results are reported.

\section{RECONFIGURABLE SWITCHING MATRIX}

It is well known that the optimal configuration for a given number of non-uniformly irradiated modules is the parallel connection. In this case, however, the output currents can exceed the allowable limits imposed by the system (i.e. inverter) [21-23]. On the other hand, it was proved that, in case of non-homogeneous irradiance, the series-connection of modules with similar irradiance produces a generated power increase. Starting from these basic issues, a fully reconfigurable Dynamic Electrical Scheme (DES) for PV generators is proposed here. The main innovations compared to existing implementations reside in the particular structure adopted, allowing a large number of module configurations. In this way, compared to existing implementations, the proposed system allows a larger increase in power output. The DES basic layout is composed by a series-connection of parallel-connected modules; this layout is usually called Total-Cross-Tie (TCT), while the series-parallel is referred as SP.

The power output of a PV system first depends on the different irradiation level, module temperature and shading. In particular, the normal case of different states of shading of the modules was considered and, under these conditions, two optimal reconfiguration algorithms have been applied to suitably enable the switching matrix. The control system uses the solar irradiances to determine the sub-optimal modules configuration following the irradiance equalization principle in the rows of the PV generator [4].

The reconfiguration system is composed of the main control module, which implements the optimal reconfiguration algorithm, and a switching matrix, which implements the layout calculated by the algorithm. The control module will be implemented on a micro-controller and will thus be adaptable to the actual physical system.

The DES switching matrix is depicted in Figure 1. It allows the reconfiguration in a single string of parallel-connected modules.

An example of its operation mode is shown in Figure 2, where $n$ is the number of the modules as well as the maximum number of rows/columns. It is worth noting that this PV 
generator topology is absolutely dynamic as regards both the number of modules that can be connected in parallel in a row and the number of rows can be connected in series. The DES allows all the configurations between the two extreme cases of all parallel-connected modules configuration (all modules stay in a row) and all series-connected modules configuration (one module per row).

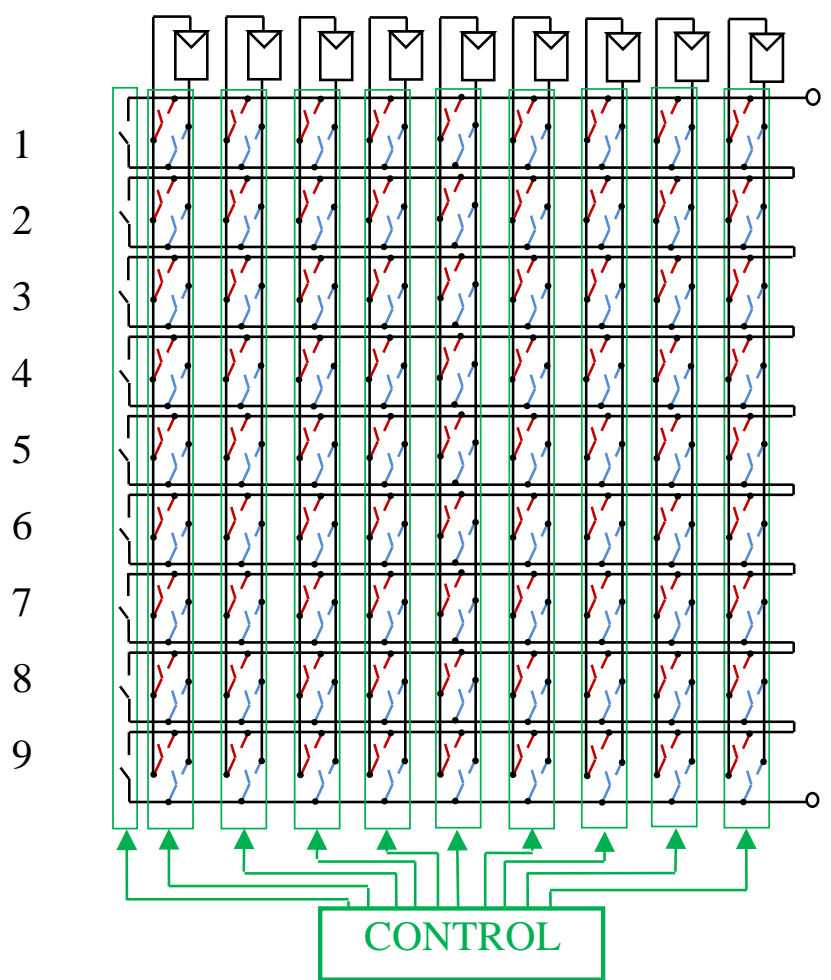

Figure 1. Dynamic Electrical Scheme switching matrix

Clearly, this flexibility is conditioned by the constraints implemented in the optimization algorithm, which calculates the minimum and maximum number of allowed rows in order to comply with the inverter input operating ranges.

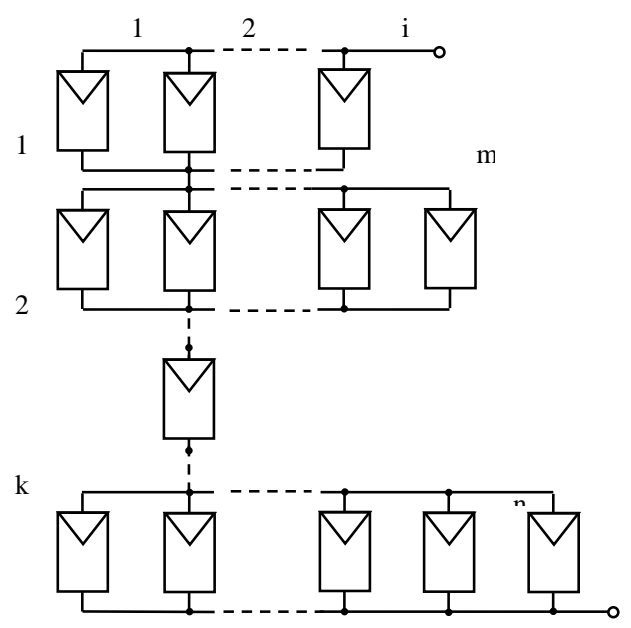

Figure 2. Generator topology 
Furthermore the algorithm also decides the maximum number of modules, that it is possible to locate in a row, depending on the maximum output current supported by connections.

The aim of the system is to relocate the shaded PV modules to maximize the power extraction from the PV generator. When some modules are shaded, they are suitably relocated within this topology in order to achieve the series-connection of the rows with similar solar irradiances, thus avoiding the damage of PV modules caused by the "hot-spot" phenomenon and maximizing the PV generator power output.

The basic structure of the switching configuration of the DES was designed considering that in a parallel-connection layout the modules order is irrelevant (Figure 3). So, in order to avoid redundant configurations, the modules can be shifted along the column only. Therefore to implement all the possible configurations, it is enough to change the module interconnections within the scheme proposed.

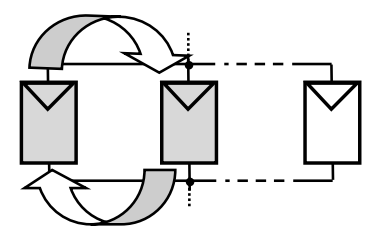

Figure 3. Redundant configurations

For instance, the system shown in Figure 1 is composed of $n=9$ reconfigurable modules; row and column indexes of the $k$-th module location are indicated with $(i, j)$ respectively. The position allowed for the considered module can range between $(1, k)$ and $(n, k)$, thus the column of module $\mathrm{k}$ will never change along the reconfiguration process. For the same system composed of $\mathrm{n}$ reconfigurable modules, the DES requires $n^{2}$ double-pole switches in order to implement the configuration calculated by the optimization algorithm. Moreover, each row of the DES is equipped with one single-pole switch, referred to as "row switches" and they are needed to guarantee the circuit continuity when there are no modules in the relevant row. It is worth noting that in [16] the switch structure requires a higher number of switches equal to $2 \cdot n^{2}$.

The flexibility of the proposed architecture has the drawback of allowing the implementation of $(n \cdot n) ! /(n !)^{\mathrm{n}}$ configurations and thus efficient optimization algorithms are needed.

\section{THE OPTIMAL RECONFIGURATION ALGORITHMS}

In a PV plant, "layout optimization" means the process of finding the best electrical connection among the modules, allowing the maximum extraction of power output from the plant. In order to find the optimal configuration, two approaches have been followed: a random search algorithm and a deterministic search algorithm. The two implemented control algorithms adopt the irradiance equalization principle which allows to equalize the available power in each row of the series string. This principle is implemented in different ways by the proposed algorithms. The random search method, with a couple of strategies to improve the efficiency of the moves, is simple to implement, efficient and fast. The main steps of the algorithm are the following:

1) Initialization;

2) Data acquisition;

3) Search for the best configuration;

4) Reallocation of modules; 
The initialization is performed during the first setup of the device.

During this step, depending on the physical PV system characteristics, the following parameters are defined:

- Number of the modules, $n$;

- Starting configuration;

- Minimum and maximum number of rows $\left(N_{R O W m i n}\right.$ and $\left.N_{R O W m a x}\right)$ that it is possible to connect in series in order to comply with the inverter input voltage operating ranges;

- Maximum current value allowed in a row.

During the acquisition step, the solar irradiance of each module and its position within a configuration are read. In the third step, the algorithm performs a random search of the best configuration following, as said, the main aim of the irradiance equalization on the row. The irradiance equalization principle for this random search method is applied. The total irradiation of each row is evaluated for each configuration tested by the control algorithm. In particular the total irradiation of the row $i$ is defined as:

$$
I_{i}=\sum_{j}^{m}=1 I_{i j}
$$

where $I_{i j}$ is the irradiance value of the module located in the row $i$ and column $j$ within the topology showed in Figure 2 and $m$ is the number of modules that are parallel connected.

For each configuration the algorithm calculates the Equalization index (E) by means of the following expression:

$$
E=\max _{i}\left(I_{i}\right)-\min _{i}\left(I_{i}\right) \quad \forall i
$$

Finally, the configuration minimizing the index $E$ is selected.

The secondary aim pursued is the smallest number of panels-switching operations compared to the starting configuration. In this way, the life span of the switches is preserved. Under the same equalization index, the configuration with the least number of switching operations to be performed is selected. The ending condition of the optimization algorithm is based on a flattening criterion. The algorithm is stopped if the irradiance equalization index does not change for a given number of iterations.

The second considered algorithm consists of the following steps:

1) Initialization;

2) Data acquisition;

3) Data ordering;

4) Modules positioning;

In the first step, the algorithm receives the same input parameter of the above-mentioned random search algorithm, except the starting configuration. After the data acquisition, modules are ordered according to decreasing irradiance values. Modules positioning, within the topology showed in Figure 2, is decided according to the irradiance equalization principle. In particular, indicating by $N_{\text {Rows }}$ the number of rows and knowing the minimum and the maximum number of rows $\left(N_{\text {ROWmin }}\right.$ and $\left.N_{\text {ROWmax }}\right)$ allowed, the algorithm tries to find the optimal configuration on the minimum number of rows $\left(N_{\text {Rows }}=N_{\text {ROWmin }}\right)$. The first $N_{\text {Rows }}$ modules of the decreasing sequence are located each in a row and then the remaining modules ordered by irradiation are located on the rows for which the sum of the irradiances of the modules already positioned is the minimum. When all modules are located and the total irradiations of rows is known, the algorithm calculates the equalization index by means of the relation (2) and stores it. Then the number $N_{\text {Rows }}$ is increased and the same procedure is repeated until $N_{\text {Rows }}$ reaches 
the maximum value $N_{R O W \max }$. Finally, the optimal configuration is the one that minimizes the equalization index.

After the optimum configuration is determined, the control module sends the open and close commands to the switching matrix, to implement the best-calculated configuration. It is worth noting that, for a given number of modules, calculation times of the deterministic search algorithm are fixed, while for the random search algorithm they change depending on the ending condition based on a flattening criterion or on prefixed number of iterations.

\section{SIMULATION OF THE PROPOSED PV SYSTEM}

In this section, the reconfiguration strategy was validated by simulations in Simulink. Under the same non-homogeneous irradiance conditions, the maximum power values supplied by a $3 \mathrm{~kW}_{\mathrm{p}} \mathrm{PV}$ generator were compared in the static configuration case and in the dynamic modules reconfiguration one. Moreover the calculation times of both proposed algorithms were calculated and compared.

In order to obtain the $3 \mathrm{~kW}_{\mathrm{p}}$ PV generator, fifteen $200 \mathrm{~W}_{\mathrm{p}}$ modules were used. Every module is realized as a series-connection of suitably arranged solar cell blocks. In table I, characteristics of the PV module used under Standard Test Conditions (Illumination level of $1000 \mathrm{~W} / \mathrm{m}^{2}$, a spectrum equivalent to $\mathrm{AM} 1.5$ and $25{ }^{\circ} \mathrm{C}$ module temperature at the test) are given.

Table 1. Electrical Characteristics of PV used Modules at $25^{\circ} \mathrm{C}$

\begin{tabular}{cclcc}
\hline$V_{M P P}$, & $I_{M P P,}$ & $P_{M M P,}$ & $V_{O C,}$ & $I_{S C,}$ \\
$\mathrm{~V}$ & $\mathrm{~A}$ & $\mathrm{~W}$ & $\mathrm{~V}$ & $\mathrm{~A}$ \\
\hline 26.01 & 7.64 & 198 & 32.40 & 8.40 \\
\hline
\end{tabular}

The DES implementation was performed connecting the "Switches blocks" of the "Simscape" library. Finally, control algorithms were implemented one by one inside the "S-Function Builder" block of the "Function \& Table" Simulink library. In the static layout, PV modules were TCT connected in a string of 5 series-connected rows, each row composed of 3 parallel-connected PV modules. The proposed system allows a complete reallocation of PV modules in a series string of whatever number of groups of parallel-connected modules, with a non-fixed number of parallel-connected modules each.

In order to estimate actual efficiency improvement by means of reconfiguration with DES, simulations in some typical real world scenarios were carried out assuming ten different irradiance conditions and registering the maximum power values, obtained both in the static case and in the dynamic one. In the first seven simulations, shading due to a change of the environmental conditions was simulated for a typical cloudy day. In this case, the irradiance level between modules was about the same. In the last three simulations, shading caused by fixed obstacles as in a typical urban environment (i.e. shadows of projected chimneys, electricity pylons and surrounding buildings) was supposed; in this case, considerable irradiance differences between irradiated modules and shaded ones were thus supposed. Numerical experimental results, in terms of obtained maximum power output values, were equal using both the algorithms and are shown in the histogram of Figure 4. 
In Figure 4, it appears that, though in all ten tests an increase of PV generator output power was obtained, there was a substantial increase in the case of shading of the modules due to fixed obstacles.

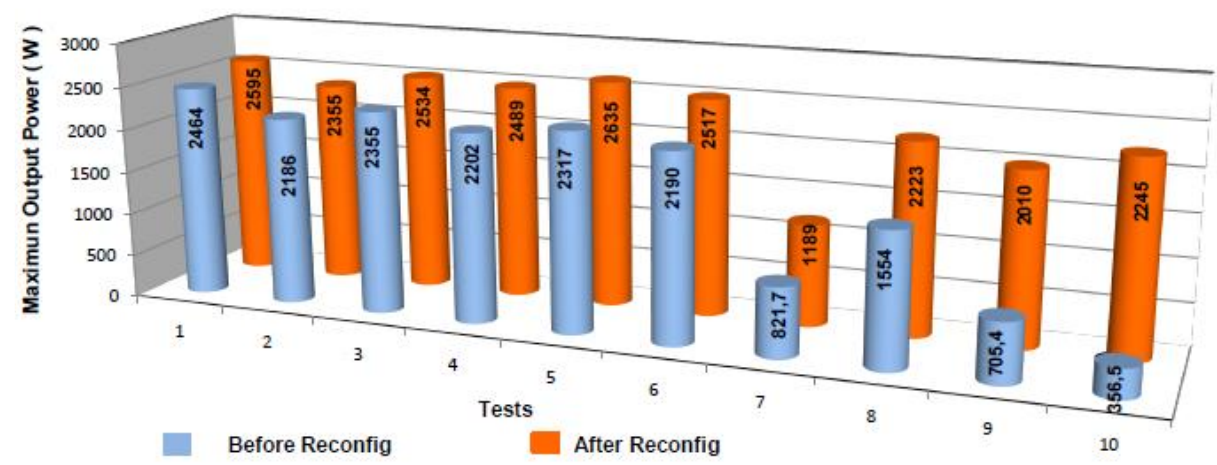

Figure 4. Graphic representation of the Maximum Power values

Power and current curves referred to the test number 8 are drawn in Figure 5, where an increase of the maximum power point after modules reconfiguration can be clearly observed. Therefore, for the above simulations of real world cases it was calculated that in case of shading due to environmental changes (first 7 simulations), the reconfiguration with DES brought a power improvement of $15.3 \%$, whereas, in case of shading due to fixed obstacles (last 3 simulations), the average output power increase was of $252.6 \%$.
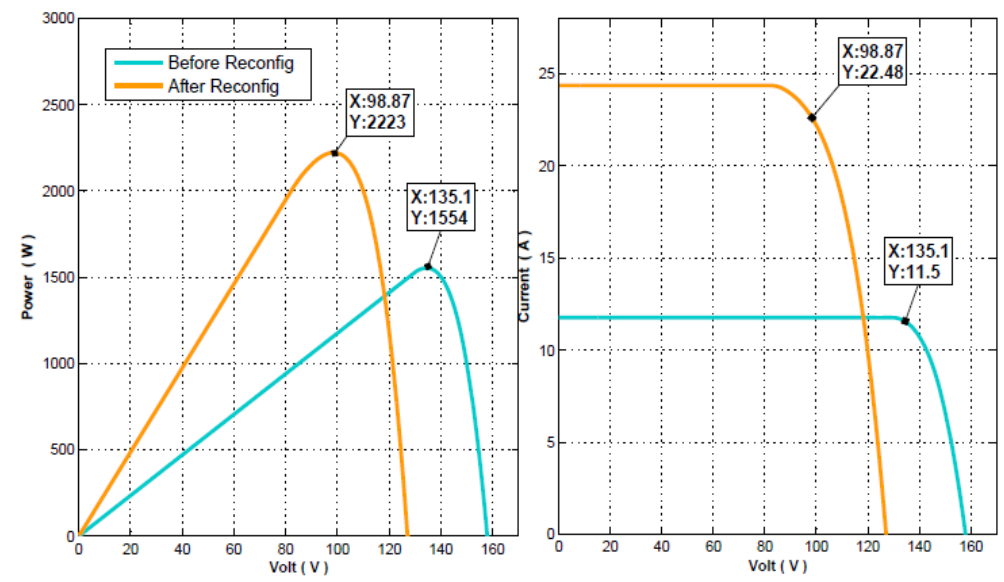

Figure 5. Power and current curves with and without dynamic reconfiguration.

As expected, experimental results in terms of calculation times were very different implementing the two algorithms. In particular, the deterministic algorithm took 0.001 seconds to obtain the optimal configuration, whereas the random search algorithm on average took 0.15 seconds.

In order to emphasize this difference Figure 6 shows, on a logarithmic scale, the trends of these times versus the number of modules.

\section{CONCLUSIONS}

In this paper the DES reconfiguration system is proposed. In our simulations, DES enabled a considerable increase in PV generator energy production under partial shading. 
In comparison to power values extracted by the PV generator in static configuration as well as with other proposed reconfiguration systems, DES allowed a satisfactory power gain both in the case of non-homogeneous irradiance due to the climatic changes (i.e. transit of clouds) and in the case of fixed obstacles. The latter is a promising result because it guarantees the potential integration of small-scale systems in urban environments, where the partial shading problem is charged with projected shadows by surrounding buildings. Moreover the proposed electrical scheme overcomes symmetrical interconnection limits. In fact, DES makes the most of the irradiance equalization algorithm, allowing rows with different number of modules.

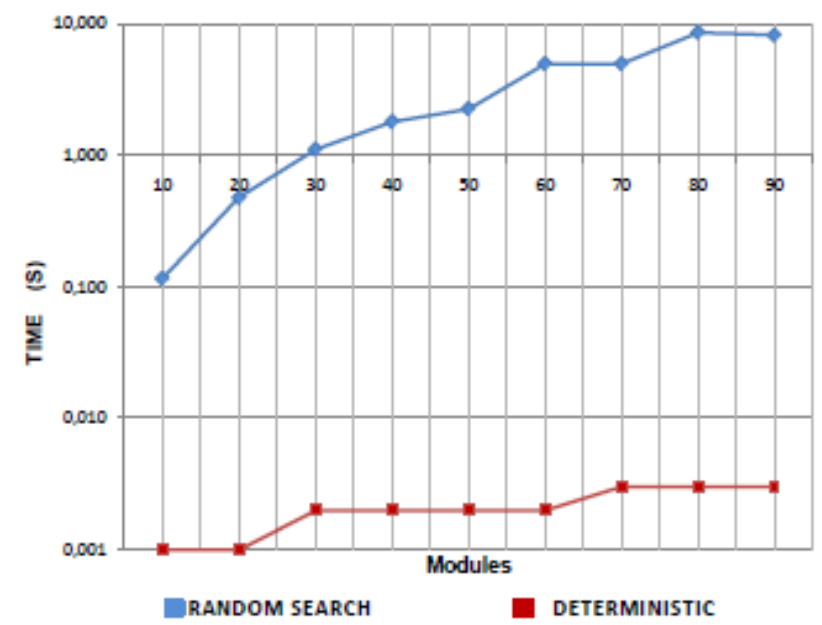

Figure 6. Calculation times versus number of modules.

The high number of possible configurations is efficiently managed by the optimization algorithms. In fact, the sub-optimal configuration is identified using limited calculation times, thus allowing adaptation to environmental change conditions in real-time. The number of switches required for the DES is still relatively high, but at least the random search algorithm maximizes the life span of the switches implementing the configuration for which the minimum number of switching operations is required. Further work will be addressed towards the implementation of DES and relevant control algorithms for larger PV generation systems.

\section{NOMENCLATURE}

MPPT - Maximum Power Point Tracking.

MPP - Maximum Power Point.

IEq-DPVA - Irradiance Equalization-Dynamic Photovoltaic Array.

DES - Dynamic Electrical Scheme.

TCT - Total-Cross-Tie.

AM 1.5 - Air Mass 1,5 - Reference Solar Spectral Irradiance.

$I$ - Irradiation

$E$ - Equalization index.

$V_{M P P}$ - Voltage in the maximum Power Point

$I_{M P P}$ - Current in the maximum Power Point

$P_{M P P}$ - Power in the maximum Power Point

$V_{O C}$ - Open Circuit Voltage

$I_{S C}$ - Short Circuit Current. 


\section{REFERENCES}

1. Kwon, J. M., Nam, K. H., Kwon, B. H., Photovoltaic Power Conditioning System with Line Connection, IEEE Trans. On Industrial Electronics, vol. 53, no. 4, pp. 1048- 1054, June 2006., http://dx.doi.org/10.1109/TIE.2006.878329

2. Kuo, Y. C., Liang, T. J., Chen, J. F., A High-Efficiency Single-Phase Three-Wire Photovoltaic Energy Conversion System, IEEE Trans. On Industrial Electronics, vol. 50, no. 1, pp. 116- 122, Feb. 2003., http://dx.doi.org/10.1109/TIE.2002.807252

3. Mutoh, N., Inoue, T., A Control Method to Charge Series-Connected Ultraelectric Double-Layer Capacitors Suitable for Photovoltaic Generation Systems Combining MPPT Control Method, IEEE Trans. On Industrial Electronics, vol. 54, no. 1, pp. 374-383, Feb. 2007., http://dx.doi.org/10.1109/TIE.2006.885149

4. Xiao, W., Dunford, W. G., Palmer, P. R., Capel, A., Application of Centered Differentiation and Steepest Descent to Maximum Power Point Tracking, IEEE Trans. On Industrial Electronics, vol. 54, no. 4, pp. 2539-2549, Aug 2007., http://dx.doi.org/10.1109/TIE.2007.899922

5. Femia, N., Petrone, G., Spagnuolo, G., Vitelli, M., Optimization of Perturb and Observe Maximum Power Point Tracking Method, IEEE Transactions on Power Electronics, vol. 20, no. 4, pp.963-973, 2005., http://dx.doi.org/10.1109/TPEL.2005.850975

6. Xiao, W., Lind, M.G.J., Dunford, W.G., Capel, A., Real-Time Identification of Optimal Operating Points in Photovoltaic Power Systems, IEEE Trans. On Industrial Electronics, vol. 53, no. 4, pp. 1017- 1026, June 2006., http://dx.doi.org/10.1109/TIE.2006.878355

7. Karatepe, E., Boztepe, M., Çolak, M., Development of a Suitable Model for Characterizing Photovoltaic Arrays with Shaded Solar Cells, Solar Energy Volume: 81, Issue: 8, August, 2007, pp. 977-992, http://dx.doi.org/10.1016/j.solener.2006.12.001

8. Woyte, A., Nijs, J., Belmans, R., Partial Shadowing of Photovoltaic Arrays with Different System Configurations: Literature Review and Field Test Results, Solar Energy 74, 217-233, 2003., http://dx.doi.org/10.1016/S0038-092X(03)00155-5

9. G., Velasco, J.J., Negroni, F., Guinjoan and R., Piqué, Irradiance Equalization Method for Output Power Optimization in Plant Oriented Grid-Connected PV Generators, 11th European Conference on Power Electronics and Applications, (EPE 05), Dresden (Germany), September 2005.

10.Auttawaitkul, Y., Pungsiri, B., Chammongthai, K., Okuda, M., A Method of Appropriate Electrical Array Reconfiguration Management for Photovoltaic Powered Car, Circuits and Systems, IEEE Asia-Pacific Conference on, 24-27 Nov. 1998 Page(s):201 - 204.

11.Salameh, Z.M., Liang, C., Optimum Switching Points for Array Reconfiguration Controller Photovoltaic Specialists, Conference Record of the Twenty First IEEE, Volume 2, 21-25 May 1990 pp.971 - 976.

12.Candela, R.; Di Dio, V., Riva Sanseverino, E.; Romano, P., Reconfiguration Techniques of Partial Shaded PV Systems for the Maximization of Electrical Energy Production International Conference on Clean Electrical Power, 2007. ICCEP '07. 21-23 May 2007 pp. $716-719$.

13.Velasco, G., Negroni, J.J., Guinjoan F., and Piqué R., Energy Generation in PV Grid-Connected Systems: A Comparative Study Depending on the PV Generator Configuration, IEEE International Symposium on Industrial Electronics, 2005. (ISIE 05). Vol. 3, pp. 1025 - 1030. Dubrovnik (Croatia), June 2005.

14.Candela, R., Riva Sanseverino, E., Romano, P., Cardinale, M., Musso D., A Dynamic Electrical Scheme for the Optimal Reconfiguration of PV Modules Under Non-Homogeneous Solar Irradiation, Applied Mechanics and Materials, vol. 197, pp. 768-777, 2012., http://dx.doi.org/10.4028/www.scientific.net/AMM.197.768 
15.Velasco-Quesada, G., Guinjoan-Gispert, F., Pique-Lopez, R., Roman-Lumbreras, M., Conesa-Roca, A., Electrical PV Array Reconfiguration Strategy for Energy Extraction Improvement in Grid-Connected PV Systems, Industrial Electronics IEEE Transactions on, Vol. 56, Issue:11 Digital Object Identifier: 10.1109/TIE.2009.2024664 Publication Year: 2009., http://dx.doi.org/10.1109/TIE.2009.2024664

16.Wilson, P., Storey, J., and Bagnall, D., Improved Optimization Strategy for Irradiance Equalization in Dynamic Photovoltaic Arrays, IEEE Transactions on Power Electronics, vol. 28, no. 6, pp. 2946-2956, 2012.

17.Cheng, Z., Pang, Z., Liu, Y., and Xue, P., An Adaptive Solar Photovoltaic Array Reconfiguration Method Based on Fuzzy Control, in Proc. 8thWorld Congr. Intell. Control Autom., Jul. 7-9, 2010, pp. 176-181.

18.Nguyen D., and Lehman, B., An Adaptive Solar Photovoltaic Array Using Model-Based Reconfiguration Algorithm, IEEE Trans. Ind. Electron., vol. 55, no. 7, pp. 2644-2654, Jul. 2008., http://dx.doi.org/10.1109/TIE.2008.924169

19.Nguyen D., and Lehman, B., A Reconfigurable Solar Photovoltaic Array Under Shadow Conditions, in Proc. 23rd Annu. IEEE Appl. Power Electron. Conf. Expo., Feb. 24-28, 2008, pp. 980-986.

20.Alahmad, M., Chaaban, M. A., Lau, S., Shi, J., Neal, J., An Adaptive Utility Interactive Photovoltaic System Based on a Flexible Switch Matrix to Optimize Performance in Real-Time, Solar Energy, Volume 86, Issue 3, Pages 951-963, March 2012., http://dx.doi.org/10.1016/j.solener.2011.12.028

21.Wright, C. G., Simulation of Solar Cell Properties with Electronic Circuit Simulation Software, Chuck Wright Consulting LLC, Round Rock, Texas.

22.Parker, G. T., Swenson, R.,: A Real World Examination of PV System Design and Performances, 31th IEEE Photovoltaic Specialists Conference, Orlando, 2005.

23.Van der Borg, N. J. C. M., Jansen, M. J., Energy Loss due to Shading in a BIPV Application, 3rd Word Conference on Photovoltaic Energy Conversion, Osaka, Japan May 11-18, 2000. 